\title{
Statement of the Asian Hypertension Society Network: the Okinawa Declaration on the unity of hypertension societies in Asian countries and regions to overcome hypertension and hypertension-related diseases
}

\author{
Yusuke Ohya $^{1} \cdot$ Kazuomi Kario $^{1} \cdot$ Hiroshi Itoh $^{1} \cdot$ Akira Nishiyama $^{1} \cdot$ Toshihiko Ishimitsu $^{1} \cdot$ Atsuhiro Ichihara $^{1}$. \\ Hisashi Kai ${ }^{1}$ - Naoki Kashihara ${ }^{1} \cdot$ Tomohiro Katsuya $^{1} \cdot$ Katsuyuki Miura $^{1} \cdot$ Masashi Mukoyama ${ }^{1} \cdot$ Satoko Nakamura ${ }^{1}$. \\ Koichi Node ${ }^{1}$ - Mitsuru Ohishi ${ }^{1}$ - Shigeyuki Saito ${ }^{1} \cdot$ Hirotaka Shibata $^{1} \cdot$ Tatsuo Shimosawa $^{1} \cdot$ Kouichi Tamura $^{1}$. \\ Kazunori Toyoda ${ }^{1}$ Mohd Arifin Mohd $\mathrm{Ali}^{2} \cdot$ Yook-Chin $\mathrm{Chia}^{2} \cdot$ Godwin Constantine $^{3} \cdot$ Erwinanto Erwinanto $^{4}$. \\ Sang Hyun $\mathrm{Ihm}^{5} \cdot$ Hsien-Li Kao ${ }^{6} \cdot$ Huynh Van Minh ${ }^{7}$ S. N. Narasingan ${ }^{8} \cdot$ Deborah Ignacia D. Ona $^{9} \cdot$ \\ Saulat Siddique $^{10} \cdot$ Apichard Sukonthasarn $^{11} \cdot$ Boon Wee Teo ${ }^{12} \cdot$ Tsolmon Unurjargal $^{13} \cdot \mathrm{Ji}^{\mathrm{G}}$-Guang Wang ${ }^{14}$. \\ Tzung-Dau Wang $^{6} \cdot$ Hiromi Raukgi ${ }^{1}$
}

Received: 1 October 2021 / Accepted: 1 October 2021 / Published online: 9 November 2021

(c) The Japanese Society of Hypertension 2021

Hypertension is the most common cause of cerebrovascular, cardiovascular, and kidney diseases, and the leading risk factor for mortality. Therefore, the management of hypertension can make an important contribution to improving healthy longevity worldwide. Asian people not only have some common characteristics in terms of culture, customs, and genetic factors, but also show diversity between areas. This affects the development and treatment of hypertension

Yusuke Ohya

ohya@med.u-ryukyu.ac.jp

${ }^{1}$ Japanese Society of Hypertension, Tokyo, Japan

${ }^{2}$ Malaysian Society of Hypertension, Petaling Jaya, Malaysia

${ }^{3}$ Sri Lanka Hypertension Society, Colombo, Sri Lanka

${ }^{4}$ Indonesian Society of Hypertension, Jakarta, Indonesia

${ }^{5}$ Korean Society of Hypertension, Seoul, South Korea

${ }^{6}$ Taiwan Hypertension Society, Taipei, Taiwan

${ }^{7}$ Vietnam Society of Hypertension, Huecity, Vietnam

${ }^{8}$ Indian Society of Hypertension, Lucknow, India

${ }^{9}$ Philippine Society of Hypertension, Pasig City, Philippines

${ }^{10}$ Pakistan Hypertension League, Karachi, Pakistan

${ }^{11}$ Thai Hypertension Society, Bangkok, Thailand

${ }^{12}$ Singapore Hypertension Society, Singapore, Singapore

${ }^{13}$ Mongolian Society of Hypertension, Ulaanbaatar, Mongolia

${ }^{14}$ Chinese Hypertension League, Beijing, China and hypertension-related diseases in each Asian country/ region. Collaborative studies in different Asian countries and regions, including Japan, have provided numerous contributions in hypertension research. Data generated by the HOPE Asia (Hypertension, Brain, Cardiovascular and Renal Outcome Prevention and Evidence in Asia) Network showed that hypertension awareness, treatment, and control rates are generally low in Asia, but these differ between Asian countries and regions [1]. The Japanese Society of Hypertension (JSH) has made several declarations and commitments, including the use of best clinical practices for hypertension management, globalization of the organization and its meetings working closely Asian and Western counterparts, and shared learning and collaboration to tackle common hypertension-related problems [2-4].

As the hypertension societies in Asian countries and regions, we consider that the next stage is to create a network for fighting hypertension and hypertension-related diseases in Asia. We strive to make contributions so that Asian researchers and practitioners can collaborate, share Asian evidence and experiences, and discuss clinical and experimental findings relevant to combatting hypertensionrelated issues in Asia.

The Okinawa islands located in East Asia have a unique geographical location and have numerous histories as one of hubs for logistics and cultures in Asia since the Age of Exploration. In these islands, we announce the "The Okinawa Declaration on the unity of hypertension societies in Asian countries and regions to overcome hypertension and hypertension-related diseases" at the 43rd Annual Scientific 
Meeting of JSH. We pledge to create and adhere to a specific action plan including the following four missions to achieve our goal of this declaration.

Hypertension societies in Asian countries and regions gathered here will:

1. Actively promote clinical and experimental investigations for hypertension research to encourage participation from researchers and practitioners from Asia.

2. Share Asian evidence to promote the establishment of standardized management of hypertension in Asia.

3. Continue to work toward developing Asian consensus guidance for the management of hypertension, using a collaborative approach and taking into account diversities within Asia.

4. Create an Asian multidisciplinary network for the management of hypertension and hypertension-related diseases.

Acknowledgements We thank Drs. Rajitha Abeysekera, Alberto A Atilano, Pairoj Chattranukulchai, Amit Gupta, Ni Made Hustrini, Tran Van Huy, Mohammad Ishaq, Dae-Hee Kim, Sirisawat Kunanon, Hung-Ju Lin, Anuj Maheshwari, Leilani B Mercado-Asis, Sungha Park, Wook Bum Pyun, Rashid Rahman, Udaya Ralapanawa, Narayanaswamy Venketasubramanian Ramani, Azhari Rosman, Weranuj Roubsanthisuk, Jinho Shin, Jam Chin Tay, Hoang Anh Tien, Namkhaidorj Tserengombo, Yuda Turana, Narsingh Verma, and Yuqing Zhang for join the working group of the Asian Hypertension Society
Network. We also thank Drs. Satoshi Hoshide, Kenichi Katsurada, Atsushi Sakima, Koichi Yamamoto, and Yuichiro Yano for organizing the session of the Asian Hypertension Society Network. The Okinawa Declaration on the unity of hypertension societies in Asian countries and regions to overcome hypertension and hypertension-related diseases was announced at the 43rd Annual Scientific Meeting of the JSH, which was held in Okinawa, Japan on October 15-17, 2021.

\section{Compliance with ethical standards}

Conflict of interest The authors declare no competing interests.

Publisher's note Springer Nature remains neutral with regard to jurisdictional claims in published maps and institutional affiliations.

\section{References}

1. Kario K, Wang JG. Could $130 / 80 \mathrm{~mm} \mathrm{Hg}$ be adopted as the diagnostic threshold and management goal of hypertension in consideration of the characteristics of Asian populations? Hypertension. 2018;71:979-84.

2. Node K, Kishi T, Tanaka A, Itoh H, Rakugi H, Ohya Y, et al. The Japanese Society of Hypertension-Digest of plan for the future. Hypertens Res. 2018;41:989-90.

3. Ito $\mathrm{M}$, Ishimitsu $\mathrm{T}$, Ichihara $\mathrm{A}$, Itoh $\mathrm{H}$, Ohishi $\mathrm{M}$, Ohkubo $\mathrm{T}$, et al. JSH statement: Kyoto declaration on hypertension research in Asia. Hypertens Res. 2019;42:759-60.

4. Nakamura S, Hasebe N, Yatabe M, Kishi T, Ishida M, Ichihara A, et al. JSH statement: Asahikawa declaration in promotion of diversity by the Japanese society of hypertension-the JSH Asahikawa declaration. Hypertens Res. 2019;42:1483-4. 